Cuadernos de Gobierno y Administración Pública

ISSN: e-2341-4839

http://dx.doi.org/10.5209/CGAP.57918

\title{
Análisis de meso y micro marcos en materia de envejecimiento activo en Galicia
}

\author{
Cristina Ares Castro-Conde* \\ Arturo de Nieves Gutiérrez de Rubalcava**
}

Recibido: 03/10/2017 / Aceptado: 04/11/2017

Resumen. Los objetivos de esta investigación son identificar barreras discursivas para la transferencia de la estrategia de envejecimiento activo de la UE y hacer recomendaciones de mejora en las políticas de envejecimiento. Se presenta un estudio exploratorio realizado en Galicia, región española con un perfil poblacional particularmente envejecido y amplias competencias en asuntos sanitarios y sociales, temas sustanciales en la estrategia europea de envejecimiento. Se emplea la palabra "marco" en el sentido de Goffman, en referencia a las ideas que permiten a un individuo conferir significado a su experiencia. Se examinan marcos de nivel macro (europeo), meso (área de políticas) y micro (actores). Se analizan datos a dos niveles: el de la comunidad epistémica regional en materia de envejecimiento activo, y el de las personas mayores activas tanto de entornos rurales como urbanos. Las técnicas de investigación empleadas son la entrevista en profundidad a expertos y los grupos de discusión con séniores.

Palabras clave: Unión Europea, políticas de envejecimiento, marco discursivo, trasferencia de políticas.

\section{Analysis of meso and micro frames on active aging in Galicia}

\begin{abstract}
This piece of research aims to identify discursive barriers to transferring the EU strategy for active aging. It also delivers policy advice on the field. This is an exploratory study conducted in Galicia, a highly aged Spanish region with extended powers on health and social issues. It applies Goffman's definition of "frame" that refers to ideas that allow people to confer meaning to their experience. It examines the macro level frame (European), the meso one (policy area) and micro frames (actors). New data were created at both meso and micro levels. The research techniques used are interviews with experts and focus groups composed of active older people living in both urban and rural areas.
\end{abstract}

Keywords: European Union, aging policies, discursive frame, policy transfer.

Sumario. 1. Introducción. 2. La estrategia de envejecimiento activo de la UE. 3. Los discursos sobre envejecimiento de la comunidad epistémica gallega (meso marco) y de los mayores activos (micro marco). 4. Conclusiones. 5. Bibliografía. 6. Apéndices metodológicos.

Cómo citar: Ares Castro-Conde, C., de Nieves Gutiérrez de Rubalcaba, A., «Análisis de meso y micro marcos en materia de envejecimiento activo en Galicia», en Cuadernos de Gobierno y Administración Pública 4-2, 191-212.

* Universidad de Santiago de Compostela.

** ONU Mujeres, Nueva York. 


\section{Introducción}

El envejecimiento es un cambio demográfico de alcance global iniciado en los países desarrollados. Su principal causa es la extensión de la esperanza de vida relacionada con mejoras en las condiciones de salud. Coadyuva a este cambio demográfico el incremento de la natalidad desde el final de la Segunda Guerra Mundial hasta casi la década de 1970 (baby boom), y el más reciente descenso de la tasa de fertilidad media por debajo del nivel de reemplazo generacional de 2,1 hijos por mujer (Nagarajan et al, 2016: 6).

Los efectos sociales y políticos del envejecimiento varían entre sociedades envejecidas. No obstante, existen elementos comunes (Naciones Unidas, 2016: 67-99). El primero de ellos es el crecimiento del porcentaje de mayores o séniores sobre el total de la población. Además, aumenta el número de "mayores jóvenes" (Torp, 2015: 8) o séniores activos, con buenas condiciones físicas y cognitivas. Igualmente, las nuevas generaciones de mayores tienden a atesorar un nivel de formación más alto que las anteriores cohortes y una mayor heterogeneidad en sus experiencias vitales (Kolb, 2014).

El envejecimiento tiene, asimismo, consecuencias en las finanzas públicas. Por una parte, incrementa las necesidades de gasto en pensiones y servicios destinados a los séniores. Por otra, cuando no es compensado con la atracción de trabajadores inmigrantes, reduce el número de adultos en edad de trabajar. Incluso, este cambio demográfico puede coincidir con una crisis de empleo y/o una contracción de las cotizaciones sociales, que contribuya a aumentar los gastos y reducir la recaudación (Boeri et al, 2001). Finalmente, el proceso de envejecimiento se está produciendo en un contexto de globalización económica que puede poner en riesgo por sí misma la estabilidad de los ingresos públicos (Held y McGrew, 2002; Higgs y Gilleard, 2015; Kohli, 2015; Phillipson, 2006; Schulz y Binstock, 2006; Torp, 2015).

Existen diferentes definiciones de "mayor". La más frecuente se refiere a la persona que ha cumplido 65 años. En función de los objetivos de la investigación o de las políticas, pueden emplearse otras edades cronológicas como 55, 60 o 75 años. Dado su componente prospectivo, este trabajo analiza personas de 55 años o más, y define distintas etapas del envejecimiento: 55 a 65 años, 66 a 75, y más de 75 .

Se emplea la palabra "marco" en referencia a las ideas que permiten a un individuo otorgar significado a aspectos de su experiencia, "algo que un actor individual puede percibir, no la organización de la estructura social" (Goffman, 1974: 13). Los discursos pueden ser intencionados, con el propósito de influir sobre las preferencias de los actores (votantes, políticos, etc.), o no intencionados, algo frecuente cuando se vuelven hegemónicos. Se distingue entre marcos de nivel macro (en este caso, el de la UE), meso (el del área de políticas) y micro (el/los de los actores).

Conviene entender la relación entre las estructuras (la normativa, las instituciones responsables de formular o ejecutar una política, sus relaciones con las organizaciones de la sociedad civil, etc.) y los actores (Cram, 2011; Falleti y Lynch, 2009; López-Santana, 2006; Lynch, 2006; Schmidt, 2002 y 2010; Schmidt y Radaelli, 2004; Thatcher y Schmidt, 2013).Los marcos, como factores mediadores del impacto de las políticas públicas, afectan no solo a la configuración de preferencias sino también a la propia definición de los problemas y a la implementación (Carstensen y Schmidt, 2016; Schmidt, 2002 y 2010; Schmidt y Radaelli, 2004). 
De hecho, el discurso es con frecuencia el factor que falta en los estudios politológicos de la continuidad y el cambio en las políticas (Schmidt y Radaelli, 2004: 207; Thatcher y Schmidt, 2013: 428). También, resulta imprescindible contextualizar los discursos, porque "la indeterminación de los resultados no reside en el mecanismo [causal, como el enmarcado (framing)] sino en el contexto" (Falleti y Lynch, 2009: 1151). "Dependiendo de la naturaleza y atributos contextuales, el mismo mecanismo causal puede dar lugar a diferentes resultados" (Falleti y Lynch, 2009: 1161).

Toda estrategia de envejecimiento activo parte de la visión de una sociedad para todas las edades, que valora y ofrece oportunidades a cualquier persona con independencia de su edad cronológica (Walker, 2002: 134). Para ello, debe combatir la discriminación en base a la edad, por ejemplo, en el mercado laboral, mantener la empleabilidad de las personas a lo largo de su vida, promover la flexibilidad en la edad de jubilación, impulsar hábitos de vida saludables o el voluntariado de los séniores, garantizar el bienestar de todos los jubilados por encima de redes de seguridad mínimas, y atender a las personas de la cuarta edad, que son las más frágiles y vulnerables (Walker y Maltby, 2012). En teoría, el modelo de envejecimiento operaría simultáneamente a nivel macro, meso y micro; y el diseño de estas políticas implicaría la coordinación de distintos departamentos (Walker y Maltby, 2012: 128).

En la UE, la media de vida tras la salida del mercado laboral por alcanzar la edad de jubilación es de 19,8 años; en España, de 21,4. Como respuesta al cambio demográfico, la Comisión Europea (1999, 2002, 2007, 2012 y 2014) ha elaborado una estrategia multidimensional de envejecimiento, que comprende aspectos relacionados con el mercado laboral, las pensiones de jubilación, la salud y el bienestar de los mayores, así como su participación social y política (Eurostat, 2011). El marco europeo sobre envejecimiento es un discurso intencionado de reacción ante las dificultades financieras del sector público para mantener el gasto social destinado a la población sénior. Trata de contener la influencia de la ideología neoliberal contraria a la idea de contrato intergeneracional, revisionista de la contribución cristianodemócrata y liberal-conservadora al Estado de bienestar (Windebank y Whitworth, 2014). Este marco, con independencia de sus condicionantes económicos y demográficos, está vinculado también a un cambio cultural más amplio, que defiende una mayor intervención de los destinatarios, así como de otros actores afectados, en las distintas fases del proceso de elaboración de políticas (Hamblin, 2013).

Los principales objetivos de este trabajo son identificar barreras discursivas para la transferencia del discurso europeo de envejecimiento a los niveles meso y micro en la UE, y hacer recomendaciones de mejora en el diseño e implementación de políticas de envejecimiento. Se presenta un estudio exploratorio que analiza datos de Galicia, Comunidad Autónoma con un perfil poblacional envejecido. ${ }^{1}$ Como el resto de regiones españolas, posee amplias competencias en asuntos sanitarios y sociales, que son nucleares en la estrategia europea de envejecimiento, y pertenece a un sistema de bienestar donde también desempeñan funciones autoridades locales, tanto provinviales como municipales. Hay que destacar además que esta Comunidad ha sido reconocida por las instituciones de la UE como una de las regiones europeas de referencia en materia de envejecimeinto activo, destacándose su labor innova-

Según datos del padrón continuo a 1 de enero de 2015, elaborado por el Instituto Nacional de Estadística, la edad media de Galicia era de 45,7 años, frente a 41,9 del conjunto de España. 
dora en la aplicación de las nuevas Tecnologías de la Información y la Comunicación (TICs). Además, el envejecimiento activo es uno de los ejes de la estrategia de innovación de la Comunidad Autónoma gallega.

Se examinan datos a dos niveles: el de la comunidad epistémica regional en materia de envejecimiento activo, y el de las personas mayores activas tanto de entornos rurales como urbanos. Para su obtención, se han conducido entrevistas en profundidad a expertos y grupos de discusión con séniores.

Las técnicas de investigación cualitativas, sobre todo las entrevistas, son cada vez más empleadas para el estudio de la vejez; pese a que sigue siendo una tarea pendiente adaptar el muestreo, la recogida de datos y su análisis a los rasgos propios de estas cohortes de edad. Los grupos de discusión resultan útiles para conocer la definición de envejecimiento de los sénior activos, e identificar estereotipos y actitudes negativas que limitan su participación social y política (Walker, 2002). Como ejemplo, merece la pena destacar que las personas de más edad rara vez se consideran "mayores", y en general no se identifican con las etiquetas que les asignan las generaciones más jóvenes (Goerres, 2009; Walker y Maltby, 1997: 17-18). Por ello, ni en tareas de investigación ni desde posiciones de responsabilidad en el diseño de políticas resulta recomendable imponer definiciones y discursos de arriba-abajo, sin observar con carácter previo las prioridades y formas de enmarcar expectativas y experiencias de los mayores.

Lo que resta del texto seguirá la siguiente estructura. A continuación, se define la estrategia de envejecimiento activo de la UE. En el apartado tres, se presentan los resultados de las entrevistas en profundidad a expertos y de los grupos de discusión con séniores. Finalmente, en las conclusiones se formulan propuestas de mejora en la elaboración de políticas de envejecimiento.

\section{La estrategia de envejecimiento activo de la $\mathrm{UE}$}

Tras las primeras tensiones en los sistemas de bienestar producidas por la crisis del petróleo, desde la década de 1970 del siglo XX, emerge con fuerza desde Estados Unidos un discurso sobre las relaciones entre generaciones ajeno al Modelo Social Europeo. Se trata de un marco contrario a la idea de equidad intergeneracional característica de los Estados de bienestar de la UE (Higgs y Gilleard, 2015a; Macnicol, 2015; Moreno, 2013).

Este discurso de inspiración neoliberal se opone a la diferenciación de etapas en el ciclo vital del individuo, en la línea del giro post-moderno (Bauman, 2007; Giddens, 1991), y a reconocer a los ciudadanos el derecho a recibir protección pública en los últimos años de su vida. Este marco tuvo éxito en Estados Unidos y en el Reino Unido en la década de 1980, y explica los primeros recortes en las pensiones y servicios públicos recibidos por los séniores en estos países.

El discurso neoliberal sobre la vejez interpreta las relaciones entre generaciones como un juego de suma cero en el que las distintas cohortes de edad compiten por recursos públicos escasos. Sostiene que los jóvenes resultan perjudicados por las transferencias de rentas a los mayores. Plantea que, dado su creciente peso en el total de los electores de un país, los séniores pueden llegar a controlar el proceso democrático para imponer sus supuestos intereses comunes, lo que convertiría las sociedades envejecidas en democracias de pensionistas (Bengtson y Putney, 2006; Biggs, 
2006; Goerres 2007 y 2009; Minkler y Robertson, 1991; Quadagno 1989; Schulz y Binstock, 2006; Vincent, 1996; Walker, 1990 y 2006).

En la UE, el ajuste de los Estados de bienestar nacionales al aumento de las personas mayores es un tema político importante porque, como se acaba de decir, afecta a la continuidad del Modelo Social Europeo de crecimiento y bienestar, sobre todo en momentos de dificultades para mantener los equilibrios presupuestarios y los niveles de endeudamiento públicos, como los vividos en algunos Estados miembros durante la Gran Crisis iniciada en 2008 en Estados Unidos.

Por este motivo, las instituciones de la Unión han dedicado el año 2012 al Envejecimiento de la Población y la Solidaridad Intergeneracional, y están promoviendo el nuevo marco sobre envejecimiento a partir de una actualización del concepto de solidaridad entre generaciones. ${ }^{2}$ La Comisión ha diseñado una estrategia multidimensional de envejecimiento activo, inspirada en la idea, original de la Organización Mundial de la Salud (2002), de añadir "vida a los años" y no solo años a la vida.

A la vista del incremento en la esperanza de vida y de la crisis de natalidad, en un contexto de restricciones presupuestarias, esta estrategia prioriza los desafíos macro, como la adaptación de las política fiscales y presupuestarias, los sistemas de pensiones y seguridad social, el mercado laboral -por la posible escasez de trabajadores en activo-, los sistemas sanitarios y de cuidados institucionalizados de larga duración -debido a la necesidad de incrementar la dotación de profesionales con formación- (Eurostat, 2011: 7). Aunque, se centra en asuntos de seguridad y bienestar, y en la participación de los mayores, haciendo referencia explícita al reto de combatir los miedos a un eventual conflicto entre generaciones por el reparto de recursos públicos, despertados por el discurso neoliberal (Comisión Europea, 1999, 2002, 2005, 2007, 2012 y 2014).

El modelo de envejecimiento de la UE busca combatir la discriminación basada en la edad biológica y las actitudes y estereotipos negativos hacia los individuos de más edad, sin cuestionar la responsabilidad pública sobre la seguridad y el bienestar de los séniores. La idea de la Comisión es reemplazar los estereotipos negativos asociados a cumplir años por imágenes positivas que enmarquen la tercera edad como una extensión de la vida activa. El propósito es facilitar la continuidad en el mercado laboral, el voluntariado sénior, y otras posibles contribuciones de los mayores al bienestar social. Asimismo, pretende activar al conjunto de la población para retrasar el deterioro físico y cognitivo y con ello la pérdida de autonomía. ${ }^{3}$

Aunque no falta quien apruebe medidas como el cambio en la edad oficial de jubilación para "reducir el porcentaje de personas de la tercera edad a golpe de bolí-

2 En el marco del Año Europeo del Envejecimiento Activo y la Solidaridad Intergeneracional, el Instituto español de Mayores y Servicios Sociales (IMSERSO) elaboró un completo Libro blanco de envejecimiento activo sobre este asunto, que integra colaboraciones de expertos de distintas disciplinas. Desde la Ciencia Política, se han comenzado a publicar también estudios de políticas de envejecimiento activo, entre los que puede destacarse el elaborado por Navarro, Egea y Alba (2016) con datos de la Comunidad de Madrid.

3 Las diferencias entre el discurso neoliberal y el de la nueva solidaridad intergeneracional promovida desde las instituciones de la UE son claras. El marco neoliberal ataca la equidad intergeneracional como valor, que es un aspecto esencial de los distintos regímenes de bienestar de los Estados miembros. Este discurso ajeno a la tradición europea está siendo promovido por intereses económicos beneficiados por los recortes en las pensiones y en los servicios públicos para mayores, así como organizaciones internacionales como el Banco Mundial, el Fondo Monetario Internacional, la Organización Mundial del Comercio o, en menor medida, la Organización para la Cooperación y el Desarrollo Económicos (Walker, 2006: 343). Por tanto, eventuales transformaciones discursivas en sentido contrario al consenso europeo sobre el Modelo Social Europeo o recortes en las políticas sociales nacionales no son atribuibles a la estrategia de envejecimiento de la UE. 
grafo" (Bond et al, 2007: 305), se ha criticado a la Comisión Europea por otorgar, en la práctica, mayor importancia a los ajustes en el mercado laboral-por ejemplo, promoviendo el retraso en la edad de jubilación-, que a los temas de esta estrategia más vinculados al bienestar de las actuales cohortes de séniores (Hamblin, 2013: 5564; Walker y Malby, 2012: 119). Sin restar importancia a esta crítica, se debe apuntar que las instituciones de la UE apenas tienen atribuciones en materia de bienestar, por ejemplo, en asuntos sanitarios, atención a la dependencia o asistencia social, asuntos que continúan siendo competencia de los Estados.

En realidad, el reto del envejecimiento para la UE consiste en adaptar sistemas de bienestar públicos en los que juegan un papel clave actores de distintos niveles de gobierno: Ministerios regionales - por ejemplo, de sanidad-, departamentos locales- como los responsables de servicios de teleasistencia-, Ministros de Finanzas, y muchos otros. Esta dispersión vertical de competencias, que además es muy variable país a país, exige un gram esfuerzo de coordinación.

En definitiva, el macro marco europeo sobre envejecimiento promueve una imagen positiva del mayor activo al tiempo que defiende la idea de protección pública del ciudadano en todas las etapas de su ciclo vital. Si se estuviese transfiriendo de arriba abajo de forma exitosa, no se hallarían ideas contrarias a la participación social y política de las personas de más edad en los discursos de nivel meso y micro. Ejemplos de ello serían expresiones como: "los séniores han ganado el derecho a permanecer ajenos a los problemas sociales y políticos"/"solo deben activarse cuando su familia, su parroquia o su partido se lo piden", "las personas que permanecen más de lo exigible en el mercado laboral o asumen labores de voluntariado están restando oportunidades de empleo a los jóvenes".

En el epígrafe siguiente, se presentan los datos de esta investigación correspondientes a la Comunidad Autónoma de Galicia.

\section{Los discursos sobre envejecimiento de la comunidad espistémica gallega (meso marco) y de los mayores activos (micro marco)}

\subsection{Meso-marco}

El marco discursivo de nivel meso sobre envejecimiento activo en Galicia se define a partir de entrevistas en profundidad a personas de la comunidad epistémica gallega en esta materia. Los resultados obtenidos se presentan en cuatro bloques temáticos: definición y seriedad del problema, concepto de envejecimiento activo, prioridades e instrumentos básicos de las políticas de envejecimiento en Galicia, y papel de las asociaciones de personas mayores y otras entidades sin ánimo de lucro en su diseño e implementación.

\subsubsection{Definición del problema}

Los expertos coinciden en conferir una importancia alta o muy alta a este tema. Se argumenta que el aumento en el porcentaje de personas de más de 55 años sobre el total de la población obliga a las Administraciones públicas a gestionar los efectos del cambio demográfico en sus ámbitos competenciales. 
Son conscientes de la heterogeneidad de las cohortes de mayores, y tienden a distinguir entre personas de 55 a 65 años, 66 a 75, y más de 75. También tienen presentes las tendencias subyacentes de aumento tanto de la esperanza de vida como de los años de vida autónoma. Apuestan por hablar de "generaciones que envejecen", así como por analizar y ajustar las medidas a las características propias de cada generación. Conocen que la cohorte que acaba de cumplir 50 años reúne más personas con estudios superiores, profesionales con altas cualificaciones y cultura política más democrática. Saben que es probable que estas personas resulten más proactivas y exigentes con las Administraciones públicas durante su vejez en comparación con las generaciones actuales de mayores, y con ello que resulta imprescindible diversificar los instrumentos de las políticas de envejecimiento activo.

La mayoría de los expertos apuestan por aumentar las medidas dirigidas a las personas de entre 51 y 55 años; incluso, a partir de los 46 , en momentos de incremento del número de desempleados de larga duración. El argumento es que resulta más fácil mantener activa a una persona que permanece en esta condición tras abandonar el mercado laboral que reactivarla posteriormente.

\subsubsection{Concepto de envejecimiento activo}

Para los expertos, envejecer activamente es sinónimo de vivir más años en buenas condiciones físicas y cognitivas. Se maneja el concepto de la Organización Mundial de la Salud, que distingue tres dimensiones en la idea de envejecimiento activo: salud, participación y seguridad.

En el meso discurso destaca el convencimiento de que las políticas de envejecimiento activo constituyen una inversión rentable, porque reducen significativamente el gasto actual y futuro en atención a la dependencia. Se emplea el argumento de la racionalización del gasto para defender que las personas mayores permanezcan el máximo número de años en su hogar.

Además, la no institucionalización se considera preferible porque permite a los séniores mantenerse en sus redes sociales tradicionales, las cuales les protegen de la soledad. Esta idea, la importancia de las relaciones personales en el bienestar del mayor, emerge igualmente en los grupos de discusión con séniores y constituye un elemento compartido relevante de los marcos de los niveles más en contacto con la realidad de la vejez. Aunque, los propios mayores activos valoran también las nuevas redes generadas fuera de sus entornos profesionales y personales tradicionales. Los apoyos afectivos entre pares son una fuente significativa de bienestar que se suma a la proveniente de los intercambios en la familia.

Todos los miembros de la comunidad epistémica coinciden en que, salvo para ocupaciones que exigen gran esfuerzo físico, lo ideal es mantenerse en el ejercicio de la profesión, reduciendo la intensidad del trabajo. ${ }^{4}$ Por ello, valoran de forma especialmente positiva medidas de envejecimiento activo en el ámbito laboral como la promoción de mayores como mentores-tutores de trabajadores jóvenes así como la introducción de procesos graduales de jubilación parcial.

$4 \quad$ En el apéndice metodológico, pueden consultarse los distintos perfiles profesionales de las personas entrevistadas pertenecientes a la comunidad epistémica gallega en materia de envejecimeinto activo. 
Las personas entrevistadas vinculadas a la Sociedad Gallega de Gerontología y Geriatría y otras pertenecientes al tercer sector, reivindican en mayor medida que otros expertos la importancia de la participación social para el bienestar de los mayores. En el entorno de esta Sociedad están presentes los conceptos de "envejecimiento exitoso" y "envejecimiento saludable", de amplio uso en gerontología (Minkler, M. y P. Fadem; Rowe, J.W. y R.L. Kahn 1987 y 1998).

El primero (successful aging) se refiere al mantenimiento de las funciones físicas y mentales, a la importancia de garantizar que las personas acumulen las reservas psicológicas y físicas necesarias para soportar experiencias de estrés en los últimos años de su vida. El "envejecimiento saludable" (healthy aging), parte de que las personas mayores, lejos de constituir una carga, son sujetos muy valiosos para la sociedad. Destaca la importancia de mantenerlas activas y hacer desaparecer la discriminación basada en la edad cronológica.

Los expertos de la Sociedad de Gerontología y Geriatría manifiestan su decepción ante las resistencias a incorporar enfoques más participativos en la elaboración de las propias políticas de envejecimiento. El acento sobre la tecnificación de los cuidados, el temor de los profesionales gerontólogos y geriatras al intrusismo profesional, y rasgos propios de anteriores cohortes de mayores, menos formadas y con una cultura menos democrática que las ahora entrantes a la vejez, explicarían las dificultades para superar la vieja concepción de la persona mayor como objeto y su substitución por otra que les confiere un rol protagonista.

\subsubsection{Prioridades e instrumentos básicos de las políticas de envejecimiento activo en Galicia}

En un contexto de escasez de recursos, los actores públicos dan prioridad a la prevención de la dependencia y al apoyo a personas con autonomía limitada. Otros expertos, sin cuestionar el carácter prioritario de estas medidas preventivas, discuten cómo perseguir este objetivo, defendiendo la asignación de más recursos públicos a la prevención de enfermedades y la detección precoz del deterioro cognitivo. Estos últimos llaman la atención sobre la escasez de datos para monitorizar los cambios en el perfil y situación de la población mayor en Galicia. Algunos proponen abrir un debato conceptual sobre la noción de dependencia. Subrayan que la definición empleada en el diseño de políticas de envejecimiento activo es discutible. Defienden que dependiente es únicamente quien necesita de otras personas para realizar alguna actividad básica y ponen en valor el papel de las nuevas tecnologías como herramientas para evitar la pérdida de autonomía.

Para la Administración autonómica es fundamental la coordinación entre los departamentos con competencias en materia de sanidad, por una parte, y servicios sociales dirigidos a mayores, por otra. Estos últimos jugarían un rol clave en la sostenibilidad del gasto sanitario. Algunos expertos echan en falta una mayor coordinación incluso en la fase de planificación de las actuaciones de ambos departamentos.

Los actores públicos se hallan inmersos además en el diseño de nuevos servicios específicos para personas con autonomía limitada, con el objetivo antes mencionado de que vivan más años en su propio hogar en condiciones satisfactorias. Muchos otros expertos valoran y están interesados en esta línea de actuación. No obstante, reivindican un mayor esfuerzo para ampliar la oferta de productos adaptados a estas 
personas. Algunos ven aquí un terreno fértil para aplicar con mayor intensidad las TICs, y donde continuar innovando. Los expertos coinciden en que las personas mayores tienen temor a las TICs hasta que aprenden a manejarlas y las hacen suyas, por lo que consideran especialmente positivas las actividades formativas en este campo mientras las cohortes de mayores lo sigan precisando.

Aunque muchos expertos valoran positivamente el papel de las Administraciones municipales en la mejora del bienestar de los séniores, apuntan también que no siempre respetan la planificación autonómica, y que consideran conveniente clarificar en mayor medida el rol de la Administración provincial. Algunos entrevistados hablan de la necesidad de una mayor transversalidad en la atención a las necesidades específicas de los séniores en los distintos niveles de gobierno. Varias personas del sector privado y sin ánimo de lucro manifiestan su preocupación por la caída de recursos públicos destinados a los mayores, que complicaría la extensión de las políticas de envejecimiento activo.

Los expertos coinciden también a la hora de subrayar la resistencia de los estereotipos asociados a la edad cronológica y/o la condición de pensionista. En relación a esto, algunos llaman la atención sobre los problemas de diseño de los programas de voluntariado para mayores. Casi nunca se pone de manifiesto la aportación que el sénior hace a la persona voluntaria más joven que le ofrece apoyo afectivo o instrumental. Como norma, en las relaciones que se establecen en estos voluntariados (sean, por ejemplo, jubilados enseñando a pintar a personas de más edad, o su idioma o costumbres a inmigrantes, o a realizar ciertas gestiones a universitarios, o juegos a los niños, etc.) el protagonismo debe recaer en la persona de más edad. Algunos expertos son más cautelosos con el voluntariado sénior argumentando que todavía existe el riesgo de reemplazo de la labor de trabajadores en activo o en edad laboral con dificultades para acceder al mercado de trabajo.

\subsubsection{Papel de las asociaciones de personas mayores y de otras entidades sin ánimo de lucro}

Entre los expertos hay consenso, asimismo, sobre la importancia de la contribución de las asociaciones de personas mayores y otras entidades sin ánimo de lucro a la promoción de la participación social de los séniores. No obstante, algunos insisten en que nunca deben substituir el trabajo de los profesionales de la geriatría u otras disciplinas en dimensiones del envejecimiento activo distintas a la de participación social.

Se detecta, igualmente, coincidencia a la hora de subrayar las dificultades que tienden a sufrir las asociaciones de mayores cuando desaparecen sus líderes tradicionales, así como sobre la necesidad de adaptar el asociacionismo sénior a las demandas de las nuevas generaciones que envejecen. Varios expertos llaman la atención sobre la importancia de mantener profesionales cualificados en las asociaciones de mayores, incluso como facilitadores de la extensión de los voluntariados séniores. Esta idea también aparece en los grupos de discusión. Los mayores prefieren participar en actividades organizadas por sus asociaciones, antes que directamente por las Administraciones públicas. Además, cabe esperar que las nuevas cohortes de mayores experimenten un rechazo mayor hacia actividades organizadas por "políticos". Por otra parte, es más probable acceder al voluntariado desde una asociación (en general, a partir de una red de personas activas), y los profesionales 
del asociacionismo sénior pueden informar y asistir técnicamente en el desarrollo de estos voluntariados.

A continuación, se presenta el discurso de los séniores activos.

\subsection{Micro-marco}

La información recabada en los grupos de discusión permite definir los micro marcos sobre envejecimiento de los mayores activos en esta Comunidad Autónoma. Las dieciséis personas seleccionadas para componer los grupos están concienciadas con la idea de envejecer activamente y participan con frecuencia en actividades organizadas por miembros de la propia comunidad epistémica gallega. Una experta con amplia experiencia en el asociacionismo de mayores colaboró en el proceso de selección de las personas participantes para garantizar que todas fuesen sobresalientes por su dinamismo. Además, la composición de los grupos permite matizar el retrato de familia de los séniores participativos, identificando diferencias relevantes en función del género, el hábitat y el nivel de formación.

Se ha conducido un grupo en un entorno más urbano, la capital de la región, Santiago de Compostela, y otro en la villa de Padrón, con representación tanto de personas que viven en el núcleo del Ayuntamiento como en sus parroquias rurales. Asimismo, se equilibraron los grupos en las variables género, formación y edad. Se contó con representación de hombres (8) y mujeres (8); urbanitas (8), personas que viven en la villa (3) y en el rural (5); con estudios superiores (7), medios o básicos (6) y que no tuvieron la oportunidad de ir a la escuela (3); entre 55 e 65 años (3), entre 66 y 75 años (8), y de más de 75 años (5).

En estas conversaciones surguieron naturalmente las aspiraciones, y las estrategias y prácticas de participación de distintas cohortes de mayores activos en diversas etapas de su envejecimiento. Resultan de especial interés sus definiciones de vejez y la identificación de algunas barreras a la participación social y política.

Para mostrar estos datos se emplean cinco bloques temáticos: concepto de persona mayor y autopercepción, motivaciones para participar en actividades de envejecimiento activo, ventajas de activarse, envejecimiento en el empleo, y participación en la sociedad.

\subsubsection{Concepto de persona mayor y autopercepción}

Las personas mayores más activas observan que a lo largo de su vida ha ido cambiando el concepto de mayor. Subrayan que en los últimos años su imagen ha mejorado, y no dudan acerca de su propia capacidad para seguir aportando a la sociedad. No obstante, consideran que todavía hay mucho margen de mejora en la articulación de su contribución, porque ellos están dispuestos a ofrecer más.

"Antes ser mayor era desaparecer de la sociedad. Hoy en día creo que realmente la sociedad entendió, bueno, en parte, que las personas mayores pueden ser muy valiosas" [Mujer, 69 años, ciudad]

Enmarcan la vejez como una continuación de su vida anterior. Todos niegan rotundamente sentirse mayores. 
“Tengo 77 años y no me considero mayor, todavía no" [Mujer, 77 años, ciudad]

"Yo creo que mientras tienes ilusión por salir, por moverte, por hacer algo con las amigas, por hacer algo, no te sientes mayor. Mayor es cuando empiezas a quedarte solo en casa" [Mujer, 60 años, villa]

"En las reuniones siempre hay alguien que dice `a mí la palabra mayor solo me la dicen cuando vengo a una reunión de la Xunta o a un congreso', y nosotros, yo por lo menos, creo que es una continuación de mi vida" [Mujer, 69 años, ciudad]

"Yo me siento tan vital en muchos momentos como cuando era más joven, salvo cuando tienes esos pequeños achaques" [Mujer, 69 años, ciudad]

"Aparte del efecto rebote en el espejo, que desmejora la imagen, yo creo que todos tenemos como mecanismo de defensa no sentirnos mayores ni física ni mentalmente, y tratar de ser coherentes en esa línea de actuación para que la vejez no se instale en tu organismo" [Hombre, 70 años, ciudad]

Destacan aspectos positivos de envejecer como: acumular experiencia y sabiduría, conocerse mejor, ganar confianza y prestar menos importancia "al qué dirán".

"Para mí ser mayor es un estado biológico, uno acumula saberes, experiencia, y empiezas a olvidar; es necesario empezar a olvidar una serie de historias que tenemos registradas, porque muchas veces pueden ser un lastre para nuestra vida ahora mismo" [Hombre, 70 años, ciudad]

Entre los aspectos negativos, reconocen que les afecta la pérdida de seres queridos, y el miedo a la soledad, a una caída y a la muerte.

"Para mí es muy triste (...) Yo, en realidad, a la edad que tengo, vivo con un poco de miedo..." [Mujer, 73 años, villa]

\subsubsection{Motivaciones para activarse}

Los mayores activos entienden la participación social, ante todo, como un medio para retrasar su envejecimiento.

"Creo que es un mecanismo de defensa (...) para mantenerte, estar en contacto, como un mecanismo para sobrevivir, (...) ¡también para salir da casa!” [Hombre, 70 años, ciudad] "Como dijo el Padre, 'de niño correr, de media edad andar, y de mayor con bastón andar también, no parar, andar'. Tiene razón, no se puede parar” [Hombre, 82 años, rural]

Dejan entrever que el mantenerse activos les exige mucha fuerza de voluntad y esfuerzo.

"Lo importante es ir llegando, no quedar temprano por el camino, hacer por llegar bien. Y hace falta poner mucho de tu parte, mucho para llegar bien" [Mujer, 72 años, ciudad]

"Hace falta tener mucho ánimo, mucha voluntad" [Hombre, 76 años, ciudad]

"La vida es un estado de ánimo. Entonces, en función de cómo tengas el ánimo, como quieras tenerlo, pues te diviertes, te relacionas, empatizas, y buscas tus propios mecanismos de defensa" [Hombre, 70 años, ciudad]

En las actividades en las que participan buscan amigos, integrarse en una red social de pares. 
"Yo digo, si me voy un día para otro sitio, me voy a un centro social, si lo hay, y ya está. Ya empiezas bien, y allí vas a hacer amistades" [Mujer, 59 años, rural]

Cuando se les pregunta por qué otras personas no son tan activas, lo atribuyen a la falta de voluntad para integrarse en un grupo, a un déficit de información sobre la oferta de actividades, e incluso al rechazo que pueden generarles aquellas vinculadas a la Iglesia o a "políticos".

\footnotetext{
"Nosotros somos gente que siempre está buscando algo que hacer, no quedarse en casa, no solo ir a la alameda; hay mucha gente que no sabe qué hacer, no tienen un apoyo o una facilidad, no tienen un conocimiento de estos sitios (...) También influye la libertad de las personas. Mucha gente dice: es que es una entidad que depende de religiosos, o de políticos; y ahí la gente escapa, y ya dice que es su libertad y que se va para la alameda (...) y después tampoco tiene ansias (...) Mucha gente no tiene esa ansia, le llega con lo que hizo" [Hombre, 74 años, ciudad]
}

Las diferencias entre hombres y mujeres son relevantes. Con independencia de que en el pasado tuviesen o no un trabajo remunerado y de su nivel de estudios, las mujeres tienden a asumir el liderazgo de los grupos de séniores activos. Además, los hombres más participativos reconocen las dificultades de otros varones para integrarse. Apuntan que no son pocos quienes comienzan a asistir a alguna actividad y la abandonan pronto. Subrayan que la mayoría de los hombres solo van a los viajes. No obstante, advierten que en los últimos años ha aumentado el número de varones participativos en las actividades semanales.

También se observan disimilitudes asociadas al tipo de hábitat. En la ciudad hay más oportunidades para mantenerse activo. Los séniores rurales argumentan que el tiempo y el coste de los desplazamientos limitan su participación, y que la distancia es una barrera casi infranqueable para quienes no conducen y dependen de familiares o vecinos para salir de su entorno. Igualmente, afirman que en el ámbito rural está "peor visto" adoptar conductas participativas más allá de la familia y vecinos.

"Vivo en una aldea y mucha gente me criticó y me dijo que estaba un poco pasada" [Mujer, 59 años, rural]

\subsubsection{Ventajas de activarse}

Los séniores activos valoran, ante todo, la oportunidad de conocer y estar en contacto con otras personas, la conversación y el cariño.

\footnotetext{
"Vienes un año a actividades y conoces a más gente que viviendo toda la vida en Padrón" [Mujer, 60 años, villa]

"Yo creo que lo más importante es charlar bis a bis, o tris a tris... es muy importante. La comunicación entre personas es muy clave, mucho. Hay mucha soledad entre las personas mayores" [Mujer, 69 años, ciudad]

"La experiencia es muy satisfactoria porque la gente es muy agradable, muy abierta en general, y encuentras nuevas amistades y te hacen la vida mucho más agradable" [Mujer, 78 años, ciudad]
} 
En menor medida, afirman que les motiva y es fuente de satisfacción seguir aprendiendo.

"Pero yo aprendí muchas cosas eh, cuando vine no sabía nada de informática y ahora hago los PowerPoint, las presentaciones y así, me defiendo, aquí aprendí mucho, ahora eso sí, aprendes de otra forma. Ahora... aprendes por hobby" [Mujer, 77 años, ciudad].

Son muy conscientes de que mantenerse activos es muy positivo para ellos, sus familias y el conjunto de la sociedad.

"Aquí, conociendo un poco a determinadas personas, puede entrar alguna que por su
soledad, o problemas en la vida, puedes tratar de ayudar. Hay gente muy preparada aquí,
que conecta enseguida con ella, la arropa, la ayuda, y procura levantarle el ánimo, iy se
lo levanta!" [Hombre, 74 años, ciudad]
"Os puedo asegurar que hay gente que vino deprimida y en este momento está muy ben,
por lo que le estamos ahorrando dinero a la seguridad social en fármacos" [Mujer, 69
años, ciudad]

\subsubsection{Envejecimiento en el empleo}

Los séniores más activos coinciden en que los 65 años son, en general, una buena edad para jubilarse y poder así "disfrutar un poco de la vida". Sin embargo, harían excepciones con las ocupaciones que exigen un esfuerzo físico mayor, para las que situarían la edad de jubilación por debajo de los 65 años, así como con las profesiones para cuyo ejercicio resulta particularmente importante la experiencia, como la medicina o la enseñanza. En estas últimas, consideran razonable mantenerse en el mercado laboral hasta al menos los 70 años.

"Trabajos generales que no sean muy de función física, a los 65 años es una edad bastante aceptable. Porque a los 65 años estando bien aún puedes disfrutar algo de la vida. Pero a los 70 igual ya es muy tarde para vivirlo" [Hombre, 74 años, ciudad]

"Yo creo que depende de la salud y del trabajo: un minero tiene que dejarlo antes, un médico puede alargarlo más. No se puede generalizar" [Mujer, 78 años, ciudad]

"Yo trabajé hasta los 70, y disfruté. No me arrepiento de estar hasta esos años. Pero bueno... puedo ser una excepción" [Mujer, 77 años, ciudad]

Valorarían muy positivamente la promoción de personas mayores como mentorestutores de trabajadores más jóvenes, así como la introducción de procesos graduales de jubilación parcial. Argumentan que esto beneficiaría el aprendizaje de los jóvenes y su propio bienestar. Insisten en que les encantarían disponer de oportunidades para poder transferir capacidades y para mantener las relaciones sociales asociadas al trabajo.

"Creo que si tienes un trabajo y lo haces con gusto, estás mejor. Primero porque ganas más (...), y la vida está más, más llena...” [Hombre, 70 años, villa]

"Incluso una vez jubilado me llamaban para saber cómo se hacía tal cosa, porque la experiencia de muchos años en una ocupación (...) Entonces, cómo hacíamos esto... Yo estaría dispuesto a seguir echando una mano, pero bueno... ahora hay una ley que te jubilas... por imperativo legal. Por eso ahora no soy trabajador, soy jubiloso" [Hombre, 77, ciudad] 


\subsubsection{Participación en la sociedad}

Los mayores participativos en Galicia ven con naturalidad ayudar a sus hijos u otros familiares, tanto dentro como fuera del hogar. No le dan importancia a esta disponibilidad. Además, reconocen que disfrutan de los nietos, sobre todo cuando pueden implicarse en sus procesos de aprendizaje.

Asimismo, muchas de estas personas realizan tareas de voluntariado. Afirman que les ha llevado a dar este paso, en primer lugar, el deseo de ayudar a alguien que lo necesita y también el de conocer gente, seguir aprendiendo y mantenerse más ocupados.

"Hay muchas personas mayores que como ella participan en voluntariado, en Santiago hay muchísimas, que ayudan a familias con alimentos y ropa, a familias en crisis, familias de clase media normal, están aumentando, que eran 2 o 3 hijos, que pierden el trabajo y tienen que acudir a esos voluntarios a pedir ayuda. Y la mayoría de quienes ayudan son personas mayores, una labor encomiable" [Mujer, 78 años, ciudad]

Quienes no participan en labores de voluntariado dicen que les falta tiempo para ello. Apuntan, igualmente, que sería necesario contar con más información sobre las opciones disponibles para ello. Las personas que viven en la zona rural ven más dificultades para convertirse en voluntarias.

Algunos de los mayores activos no voluntarios destacan que se puede ayudar informalmente de múltiples maneras y argumentan que, en una sociedad donde muchas personas se sienten solas, una contribución muy valiosa es dedicar tiempo a escuchar a otros.

"Esa función de parar, escuchar y hablar es una función que practicamos ahora con las nuevas tecnologías, que el correo electrónico fue una revolución para el mundo, y hace unos años había un anuncio de Telefónica, que a mí no me gusta Telefónica, ni soy cliente, pero que cuando anunciaban móviles, el mensaje era: 'lo importante es poder hablar'. Yo creo que esa es la clave, no solo de la gente que somos un poco mayores, sino de la gente en general" [Hombre, 70 años, ciudad]

\subsubsection{Valoración de las políticas de envejecimiento y expectativas de futuro}

Los mayores más activos valoran positivamente que existan políticas de envejecimiento activo. Incluso, manifiestan una gran preocupación por la posibilidad de que las dificultades financieras impliquen recortes en la promoción de la autonomía personal y de su oferta de actividades. Advierten de que, si aumenta el precio de las mismas para ellos, muchos séniores activos, sobre todo quienes con su pensión ayudan a hijos y nietos, dejarían de participar. Insisten en que, para valorar su capacidad de pago, se tengan en cuenta no solo su pensión sino sus obligaciones familiares.

Estos séniores creen que es importante seguir haciendo un esfuerzo para mantener y ampliar residencias, centros de día y en general todas las instalaciones públicas donde conviven mayores. De estos centros, e incluso de los servicios de teleasistencia, valoran no solo la atención profesional sino particularmente el hecho de que les permiten estar en contacto con otras personas. 
"No es lo mismo que te vaya una persona de ayuda a domicilio a ayudarte a ducharte, a asearte y la cama, a que tú puedas estar con otra gente que se encuentra en tus mismas condiciones con las que puedes compartir un rato, un tiempo...” [Mujer, 60 años, villa]

No obstante, se muestran desencantados y esperan poco de las Administraciones públicas. En cambio, coinciden en poner en valor el papel de las asociaciones de mayores y otras organizaciones del tercer sector, con las que se siente más identificados.

“(...) agrupaciones en las que uno puede caer y encontrar un ámbito que le ayuda en lo que necesite, que lo escuche... Yo de la Administración no espero nada(...) Si la Administración tuviese interés, debería crear un banco de personas con saberes (...) para acudir a ellas" [Hombre, 70 años, ciudad]

Todavía con relación a las dificultades financieras del sector público, los séniores dejan entrever su temor a una bajada de las pensiones de jubilación y en general al deterioro del Estado de bienestar.

"El futuro se ve un poco negro" [Mujer, 60 años, villa]

"El semáforo está amarillo" [Hombre, 82 años, rural]

\section{Conclusiones}

En este artículo se han analizado los marcos de nivel meso (área de políticas) y micro (mayores activos) sobre envejecimiento activo en Galicia. Estos discursos contienen elementos presentes en el macro marco definido por la Comisión Europea, pero también otros rasgos que entran en contradicción con este. Así, se han identificado barreras discursivas contra las que chocan los mayores activos y la propia transferencia de la estrategia europea de envejecimiento activo en esta región.

Para terminar, a partir de la evidencia empírica de los grupos de discusión con personas mayores activas y las entrevistas en profundidad a miembros de la comunidad epistémica gallega en la materia, se proponen las siguientes recomendaciones de mejora en el diseño e implementación de políticas de envejecimiento.

Se sugiere evitar el término "envejecimiento", dado que las propias personas mayores no se identifican con estas políticas porque no se consideran mayores.

La clave para el bienestar de los séniores puede residir en el tejido y dinamización de redes sociales de prevención de la soledad. La comunicación institucional y el lenguaje administrativo podría girar, entonces, en torno a la idea de combatir la soledad.

Resulta prioritario realizar un mayor esfuerzo para eliminar la discriminación asociada a la edad de todos los ámbitos, y en particular del mercado laboral.

Se espera que las nuevas generaciones de jubilados demanden una mejor atención de las Administraciones públicas, cuenten con más varones activos (las mujeres van por delante en esta materia) y estén más motivadas para seguir contribuyendo a la sociedad. Cabría involucrar más a las personas mayores desde una fase temprana de la elaboración de políticas y apostar por fórmulas participativas.

Es recomendable graduar el nivel de participación a cada persona, y ampliar las posibilidades o niveles de implicación para satisfacer las expectativas de los más exigentes. El primer paso es mantenerse activo. Luego, algunos séniores se convertirán 
en voluntarios. Los profesionales de las asociaciones de mayores están en condiciones de jugar un papel clave como porteros y facilitadores del tránsito desde actividades menos exigentes al voluntariado, informando, asistiendo técnicamente y motivando.

Incluso en las políticas de atención a la dependencia, el mayor debería ser siempre el protagonista. El resto de actores se considerarán secundarios y su rol consistirá en habilitar y potenciar, no en dirigir o substituir.

Resulta imprescindible fomentar conductas participativas y el compromiso cívico desde la infancia. Activar o reactivar a las personas de más edad ofrece pocas garantías sin una formación previa y temprana.

Las medidas dirigidas a los mayores deben ajustarse a las características propias de cada generación y etapa de envejecimiento (de 46 a 55 años, de 56 a 65 años, de 66 a 75 años, y mayores de 75 años). Conviene, en general, adaptarlas las medidas a los públicos objetivos concretos, definiendo con precisión los destinatarios de cada programa.

Se recomienda contar con equipos de trabajo interdepartamentales y multidisciplinares. Únicamente para perseguir los objetivos de prevención de la dependencia, se requiere un enfoque multidimensional, en el que tengan presencia la $\mathrm{I}+\mathrm{D}+\mathrm{i}$ en el ámbito de la gerontología y la geriatría, la promoción de mayores como mentorestutores de trabajadores más jóvenes, el mantenimiento del poder adquisitivo de las personas jubiladas, el apoyo a la profesionalización del asociacionismo de mayores o la organización de actividades culturales con precios públicos reducidos.

Finalmente, se requiere una mayor coordinación entre niveles de gobierno, y desarrollar la cultura de la evaluación, poniendo en marcha equipos interdepartamentales de seguimiento y mejora continua.

\section{Bibliografía}

Bauman, Z. (2007). Liquid times: Living in an age of uncertainty. Polity, Cambridge.

Bengtson, V.L. y Putney, N. M. (2006). "Future 'conflicts' across generations and cohorts?", en Vincent, J.A., Phillipson, C.R.y Downs, M. The futures of old age. Sage, Londres, pp. 20-29.

Biggs, S. (2006). "Ageing selves and others: Distinctiveness and uniformity in the struggle for intergenerational solidarity", en Vincent, J.A., Phillipson, C.R.y Downs, M. The futures of old age. Sage, Londres, pp. 109-116.

Boeri, T., Börsch-Supan, A., Brugiavini, A., Disney, R., Kapteyn, A y Peracchi, F. (2001). Pensions: More information, less ideology. Assessing the long-term sustainability of european pension systems: Data requirements, analysis and evaluations. Springer, New York.

Bond, J., Dittmann-Kohli, F., Westerhof, G.J y Peace, S. (2007). "Ageing into the future", en Bond, J., Peace, S., Dittmann-Kohli, F y Westerhof, G. Ageing in society. Sage, Londres, pp. 296-308.

Carstensen, M.B. y Schmidt, V.A. (2016). "Power through, over and in ideas: Conceptualizing ideational power in doscursive institutionalism", en Journal of European Public Policy, vol. 23, n 3, pp. 318-337.

Comisión Europea (1999). Towards a Europe for all ages-Promoting prosperity and intergenerational solidarity, COM (1999) 221 final. 
Comisión Europea (2002). Europe's response to world ageing: Promoting economic and social progress in an ageing world. A contribution of the European Commission to the 2nd world assembly on ageing, COM (2002) 143 final.

Comisión Europea (2005). Libro Verde "Frente a los cambios demográficos, una nueva solidaridad entre generaciones", COM (2005) 94 final.

Comisión Europea (2012). Comunicación de la Comisión al Parlamento Europeo y al Consejo "Llevar adelante el Plan Estratégico de Aplicación de la cooperación de innovación europea sobre el envejecimiento activo y saludable”, COM (2012) 83 final.

Comisión Europea (2014). The 2015 ageing report. Underlying assumptions and projection methodologies, Bruselas.

Cram, L. (2011). "The importance of the temporal dimension: new modes of governance as a tool of government", en Journal of European Public Policy, vol. 18, $\mathrm{n}^{\mathrm{o}} 5$, pp. 636-653.

Eurostat (2011). Active ageing and solidarity between generations. A statistical portrait of the European Union 2012. Luxemburgo: Oficina de Publicaciones de la UE.

Eurostat (2015). Demography report, analytical web note, 3. Oficina de Publicaciones de la Unión Europea, Luxemburgo.

Falleti, T. y Lynch, J. (2009). "Context and causal mechanism in political analysis", en Comparative Political Studies, vol. 42, no 9, pp.1143-1166.

George, L. (2006). "Perceived quality of life", en Binstock, R.H. y George, L.K. Handbook of the aging and the social sciences. Academic Press, Ámsterdam, pp. 320-336.

Giddens, A. (1991). Modernity and self-identity. Polity Press, Cambridge.

Goerres, A. (2007). Can we reform the welfare state in times of 'Grey' Mmajorities? The myth of an electoral opposition between younger and older voters in Germany. Max Planck Institute for the Study of Societies. Working Paper 07/05.

Goerres, A. (2009). The political participation of older people in Europe. The greying of our democracies. Palgrave, Basingstoke.

Goffman, E. (1974). Frame analysis. An essay on the organization of experience. Harper \& Row, Nueva York.

Hamblin, K.A. (2013). Active ageing in the European Union. Policy convergence and divergence. Palgrave, Houndmills.

Held, D. y McGrew, A. (2002). Governing globalization: Power, authority and global governance. Polity Press, Cambridge.

Higgs, P. y C. Gilleard (2015). "Generational Justice, generational habitus and the 'problem' of the baby boomers", en Torp, C. (ed.). Challenges of aging. Pensions, retirement and generational justice. Palgrave, Basingstoke, pp. 251-263.

Instituto de Mayores y Servicios Sociales. (2011) Libro blanco del envejecimiento activo. Ministerio de Sanidad, Política Social e Igualdad, Madrid.

Kohli, M. et al. (2010). "Linkages among adult family generations: Evidence from comparative survey research", en Heady, P. y Kohli, M. (ed.) Famili, kinship and state in contemporary Europe, vol 3: Perspectives on theory and policy. Campus, Frankfurt, pp. 195-220.

Kohli, M. (2015). "Generations in aging societies: Inequalities, cleavages, conflicts" en Torp, C. (ed.). Challenges of aging. Pensions, retirement and generational justice. Palgrave, Basigstoke, pp. 265-288. 
Kolb, P. (2014). Understanding aging and diversity. Routledge, Nueva York.

López-Santana, M. (2006). "The domestic implications of European soft law: Framing and transmitting change in employment policy", en Journal of European Public Policy, vol. 13, n 4, pp. 481-499.

Lynch, J. (2006). Age in the Welfare State. The origins of social spending on pensioners, workers, and children. Cambridge University Press, Cambridge.

Lynch, J. (2015). "Age politics and pension systems development and reform", en Torp, C. (ed.). Challenges of aging. Pensions, retirement and generational justice. Palgrave, Basingstoke, p. 49-60.

Macnicol, J. (2015). Neoliberalising old age. Cambridge University Press, Cambridge.

Minkler, M. y Robertson, A. (1991). "The ideology of age/race wars: Deconstructing a social problem", en Ageing and Society, $\mathrm{n}^{\circ}$ 11, pp. 1-23.

Minkler, M. y Fadem, P. (2002). "Successful aging: A disability perspective”, en Journal of Disability Policy Studies, n 12, pp. 229-235.

Moreno, L. (2013). La Europa asocial. Península, Barcelona.

Naciones Unidas (2003). Declaración politica y Plan de Acción Internacional de Madrid sobre el Envejecimiento. Naciones Unidas, Nueva York.

Naciones Unidas. (2016). World population ageing 2015 (ST/ESA/SER.A/390). Disponible en:http://www.un.org/en/development/desa/population/publications/pdf/ageing/WPA2015_Report.pdf, 09/01/2017.

Nagarajan, R., Teixeira, A y Silva, S. (2016). "The impact of an ageing population on economic growth: An exploratory review of the main mechanisms", en Análise Social, vol. 51, pp. 218, 4-35.

Navarro, C., Egea, A y Alba, S. (2016). Políticas públicas locales en Ayuntamientos de la Comunidad de Madrid (educación, mayores y medio ambiente). Instituto de Derecho Local de la Universidad Autónoma, Madrid.

Organización para la Cooperación y el Desarrollo Económico. (1988). Ageing populations - The social policy implications. OCDE, París.

Organización para la Cooperación y el Desarrollo Económico. (1998). Maintaining prosperity in an aging society. OCDE, París.

Organización para la Cooperación y el Desarrollo Económico. (2001). Aging and income. OCDE, París.

Organización Mundial de la Salud. (2002). Active ageing: A policy framework. Disponible en: http://apps.who.int/iris/bitstream/10665/67215/1/WHO_NMH_ NPH_02.8.pdf, 26/09/2016.

Phillipsō, C. (2006). "Ageing and Globalization", en Vincent, J.A.; C.R. Phillipson y M. Downs. The Futures of Old Age (pp. 201-207). Londres: Sage.

Quadagno, J. (1989). "Generational equity and the politics of the Welfare State", en Politics and Society, no 17, pp. 353-376.

Rowe, J.W. y Kahn, R.L. (1987). "Human aging: Usual and successful", en Science, vol. 237, no 4811, pp. 143-149.

Rowe, J.W. y R.L. Kahn (1998). Successful aging. Pantheon Books, Nueva York.

Schmidt, V.A. (2002). "Europeanization and the mechanics of economic policy adjustment", en Journal of European Public Policy, vol. 9, nº 6, pp. 894-912.

Schmidt, V.A. (2010). "Taking ideas and discourses seriously: Explaining change through discursive institutionalism as the fourth 'new institutionalism',, en European Political Science Review, vol. 2, nº 1, pp. 1-25. 
Schmidt, V.A. y Radaelli, C.M.(2004). "Policy change and discourse in Europe: Conceptual and methodological issues", en West European Politics, vol. 27, $\mathrm{n}^{\mathrm{o}}$ 2, pp. 183-210.

Thatcher, M. y V.A. Schmidt (2013). "Conclusion: Explaining the resilience of neoliberalism and possible pathways out", en Schmidt, V.A. y Thatcher, M. Resilient liberalism in europe's political economy. Cambridge University Press, Cambridge, pp. 403-429.

Torp, C. (2015). Challenges of aging. Pensions, retirement and generational justice. Palgrave, Basingstoke.

Schulz, J.H. y Binstock, R.H.(2006). Aging nations: The economics and politics of growing older in America. Praeger, Westport.

Sidorenko, A. y A. Walker (2004). "The Madrid International Plan of Action on ageing: From conception to implementation", en Ageing and Society, vol. 24, $\mathrm{n}^{\circ} 1$, pp. 147-165.

Vincent, J. (1996). "Who's afraid of an aging population? Nationalism, the free market and the construction of old age as an issue", en Critical Social Policy, vol. 16, pp. 3-26.

Vincent, J.A., Phillipson, C.R y Downs, M. (2006). The futures of old age. Sage, Londres.

Walker, A. (1990). “The economic 'burden' of aging and the prospect of intergenerational conflict", en Ageing and Society, vol. 10, n 4, pp. 377-396.

Walker, A. (2006). "Aging and politics: An international perspective", en Binstock, R.H. y George, L.K. (ed.). Handbook of the aging and the social sciences. Academic Press, Ámsterdam, PP. 339-359.

Walker, A. y Naegele, G. (2009). "Major policy challenges of ageing societies: Britain and Germany compared", en Walker, A. y Naegele, G. (ed.). Social policy in ageing societies. Britain and Germany compared. Palgrave, Basingstoke, pp. 1-21.

Walker, A. y T. Malby (1997). Ageing Europe. Open University Press, Buckingham. Westerhof, G.J. y Tulle, E. (2007). "Meaning of ageing and old age: Discursive contexts, social attitudes and personal identities", en Bond, J., Peace, S., DittmannKohli, F y Westerhof, G. Ageing in society. Sage, Londres, pp. 235-254.

Windebank, J. y Whitworth, A. (2014). "Social Welfare and the ethics of austerity in Europe: Justice, ideology and equality", Journal of Contemporary European Studies, vol. 22, n 2, pp. 99-103.

\section{Apéndices metodológicos}

Apéndice metodológico I: perfil de las personas entrevistadas, y guión de las entrevistas en profundidad a miembros de la comunidad epistémica regional en materia de envejecimiento activo

Esta entrevista forma parte de un estudio que pretende analizar la evolución en la última década de las políticas de envejecimiento activo en Galicia a nivel autonómico y local. Examina asimismo los procesos participativos de cada generación que envejece y etapa del proceso de envejecimiento. El principal objetivo es identificar futuras áreas de intervención para informar la elaboración de políticas en la materia. 
Se considera que su participación como experto entrevistado es importante debido a su conocimiento en profundidad de esta área de políticas y la relevancia de sus percepciones y expectativas. El siguiente listado de preguntas es únicamente un guión de temas sobre los que nos gustaría conversar con usted.

P1. ¿Desde cuándo está familiarizado con el concepto de envejecer activamente?

P2. ¿De dónde proviene su interés por el envejecimiento activo?

P3. ¿Qué aspectos de las políticas de envejecimiento activo funcionan mejor en Galicia, y por qué?

P4.¿Qué elementos deberían eliminarse, y por qué?

P5. ¿Qué aspectos de estas políticas convendría mejorar, y por qué?

P6. ¿Qué programas o actividades podrían añadirse, y por qué le parecen importantes?

P7. ¿Cómo cree que evolucionarán las políticas de envejecimiento activo en Galicia, y cómo afectarían estos cambios a su entidad?

P8. De acuerdo con su experiencia, ¿para qué resultan más útiles a las personas mayores las nuevas tecnologías de la información y las comunicaciones (Internet, ordenador, teléfono móvil de nueva generación, etc.)?

P9. ¿Qué tiene comprobado usted que verdaderamente funciona para activar la participación en la sociedad de las personas mayores?

P10. ¿Cree que la valoración social de los mayores es satisfactoria? En caso negativo, ¿qué medidas recomendaría para reducir los estereotipos asociados a la edad?

Los perfiles de las personas entrevistadas son los siguientes:

- Un responsable político de la Administración autonómica en materia de política social (Entrevista realizada el jueves día 14 de noviembre de 2013, a las 10:00 horas, en el Edificio Administrativo San Caetano, s/n, Santiago de Compostela)

- Un responsable técnico de la misma área (Lunes 11 de noviembre, 13:30 horas, Edificio Administrativo San Caetano, s/n, Santiago de Compostela)

- Dos profesionales del sector privado:

- Martes 5 de noviembre, 19:00 horas, Vigo

- Viernes 15 de noviembre, 10:00 horas, A Coruña

- Cuatro profesionales del sector sin ánimo de lucro:

- Martes 5 de noviembre, 10:30 horas, Santiago de Compostela

- Viernes 8 de noviembre, 11:00 horas, A Coruña

- Viernes 22 de noviembre, 12:00 horas, Santiago de Compostela

- Martes 26 de noviembre, 10:00 horas, A Coruña

\section{Apéndice metodológico II: guión de los grupos de discusión con séniores activos}

Presentación del grupo y de los participantes (Tiempo estimado: 5 minutos)

Buenos días. Mi nombre es ... Hoy también nos acompaña mi compañera ... En primer lugar, me gustaría agradecer a cada uno de ustedes, tanto en nuestro nombre como en el de la USC, su participación en este grupo de discusión. 
Como ya les han informado, la USC está llevando a cabo un estudio en el marco del proyecto europeo "Envejecimiento + ACTIVO" financiado por el Fondo Europeo de Desarrollo Regional (FEDER) dentro del Programa Operativo de Cooperación Transfronteriza España-Portugal, .... El proyecto se estructuró en dos fases, una cuantitativa y otra cualitativa, en la que se integra este grupo de discusión. La parte del estudio en la que ustedes participan quiere capturar las opiniones y debates vivos en la sociedad con respecto al bienestar de las personas mayores de 55 años. Se trata de recoger elementos de los discursos que articulan los debates públicos en torno a la vida cotidiana de los séniores, la influencia de las Administraciones públicas en su satisfacción vital, y sus expectativas de futuro.

Los grupos de discusión reúnen a un número reducido de personas para debatir informalmente de manera abierta, sin cuestionarios ni preguntas, salvo los bloques temáticos que iré poniendo sobre la mesa. Por tanto, mi participación será mínima. Se trata de que ustedes expresen sus opiniones sin reglas ni turnos de palabra, salvo el respeto a las normas de educación mínimas. También me gustaría aclarar que en esta charla no se trata de averiguar quién sabe más de los temas. No hay respuestas correctas ni erróneas, buenas ni malas. Nos interesan sus impresiones expresadas con libertad. En este sentido, pese a que el grupo será grabado para facilitar el tratamiento de la información, en ningún caso se identificará a la persona que emita una opinión. Nos interesa el discurso general del grupo, y el tratamiento de la información será agregado y anónimo, de acuerdo con la legislación sobre protección de datos. Por ello, y para que sus experiencias mejoren el estudio, les rogamos que se expresen con sinceridad y en el idioma (gallego o castellano) en el que se sientan más cómodos.

Para comenzar, nos gustaría que cada uno de ustedes se presentase brevemente (nombre, a qué se dedica o en qué ha trabajado, etc.). Como ya les he comentado, no hay turnos de palabra ni ninguna otra regla especial.

Bloque temático 1. Percepción sobre el concepto y la imagen de los mayores (Tiempo estimado: 30 minutos)

- Entrando en materia, me gustaría que me dijesen ¿qué es eso de ser mayor...? Ya saben, ¿a qué nos referimos cuando hablamos de personas mayores?

- (Solo si este dato no ha salido antes) ¿A qué edad consideran ustedes que se es mayor?

- Y más específicamente, se está hablando mucho últimamente de la edad de jubilación, ¿a partir de qué años creen ustedes que una persona debe dejar de trabajar?

- Hablando ahora en general, ¿consideran que la imagen de los mayores en la sociedad se ajusta a la realidad?, es decir, ¿existen diferencias entre cómo se ven a sí mismos los mayores y cómo los ve la sociedad? (De ser así) ¿Qué diferencias?

- Y, teniendo en cuenta lo que acabamos de comentar, ¿podrían decirme si ustedes se sienten mayores? 
Bloque temático 2. Vida cotidiana (Tiempo estimado: 60 minutos)

- Hablando un poco más sobre ustedes mismos y lo que hacen cada día, ¿creen que en su día a día tienen oportunidades suficientes para desarrollar sus capacidades y emplear sus conocimientos? ¿Por qué?

- ¿Y en sus rutinas diarias encuentran alguna limitación? (Si es así) ¿Cuáles les preocupan más?

- En general, ¿creen que lo que hacen a diario es útil o provechoso para otras personas?

- Alguno de ustedes se ocupa todos o algún día de la semana de sus nietos u otros familiares? (Si es así) ¿Les gusta hacerlo?

- ¿Conocen a alguna persona de 55 años o más que esté preocupada por llegar a fin de mes? (Si es así) ¿Cuáles dirían ustedes que son los motivos (sueldo o pensión baja, ayudan mucho a sus hijos y nietos, la propia crisis...)?

- ¿ Actualmente ustedes están satisfechos con su vida? ¿Mucho?

- ¿Qué expectativas tienen a medio y largo plazo? ¿Creen que para la gente mayor las cosas irán a mejor o a peor? ¿Por qué?

Bloque temático 3. Participación social y política (Tiempo estimado: 25 minutos)

- ¿Alguno de ustedes colabora con organizaciones de voluntarios o de ayuda a personas que lo necesitan? (Si es así) ¿Qué motivaciones les llevaron a hacerlo?

- ¿Les gustaría participar más en política? (Si es así) ¿Cómo: militando en un partido, colaborando en un movimiento social, siendo concejal, etc.?

- En otro orden de cosas, ¿qué aspectos mejorarían ustedes de los programas y medidas que conocen de su Ayuntamiento, de la Diputación o de la Xunta de Galicia dirigidas a las personas de 55 años o más?

- En sentido contrario, ¿cuáles de estas actividades les interesan y aportan más? 